\title{
Microcontroller Based Wireless Temperature And Heart Beat Read-Out
}

\author{
Nisha singh ${ }^{1}$, Sr. Asst. Prof. Ravi Mishra ${ }^{2}$ \\ ${ }^{1}$ (ME II Year VLSI Design, Department Of EC, SSCET, Gunwani, Bhilai Nagar (C.G), India) \\ ${ }^{2}$ (Sr. Asst. Prof. Department Of EEE, SSCET, Gunwani, Bhilai Nagar (C.G), India)
}

\begin{abstract}
In this paper, we propose a Simple Wireless transmission System using common approach Sensor Platform called The wireless based Patient Sensor platform (WSP, Sensor Node) which has remote access capability. The goals of the WSP are to establish: Standard sensor node (System on module), a common software .The proposed platform architecture (Sensor Node) offers flexibility, easy customization for different vital parameter collecting and sending. An prototype has been established based on wireless communication channel. Wireless lan (IEEE .802.15.4)) has been used as communication channel on our prototype (Sensor node). Desire sensor information (vital parameter) can be viewed remotely, and also vital parameter can be adjusted to meet demand.
\end{abstract}

Keywords: Microcontroller, Heart Rate sensor, Temperature sensor, RF-Transmitter and Receiver with LCD.

\section{INTRODUCTION}

Microcontroller Based Wireless Temperature And Heart Beat Read Out suitable for operation in a small office/home environment. This system is easy to operate, with Visual LCD. Many individuals and organizations may, for various reasons, wish to use electronic surveillance techniques at some time or another. The idea is to use off-the-shelf RF Tx/Rx modules. The weather keeps us continually occupied. Some people have even made it their profession. At home too, we like to measure all kinds of things related to our climate. That is why weather stations are available in all types and sizes. If we want to know the temperature inside and outside then purpose-built indoor/outdoor thermometers are available. In the past the outside sensor of these weather stations was connected with a wire, it is now fairly standard to use RF transmission for this data. This Wireless transmitters units usually make use of the $315-\mathrm{MHz}$ band. These modules, once a rare commodity, are now widely and cheaply available. In this particular discussion, we shall be using ASK (Amplitude Shift Keying) based TX/RX pair operating at $315 \mathrm{MHz}$. The transmitter module accepts serial data at a maximum of $2400 \mathrm{bps}$. They are directly interfaced to a microcontroller. At the RX end, the receiver microcontroller receives the signal via the RF receiver module, decodes the serial data and reproduces the original data in the temperature and Heat Beat format.

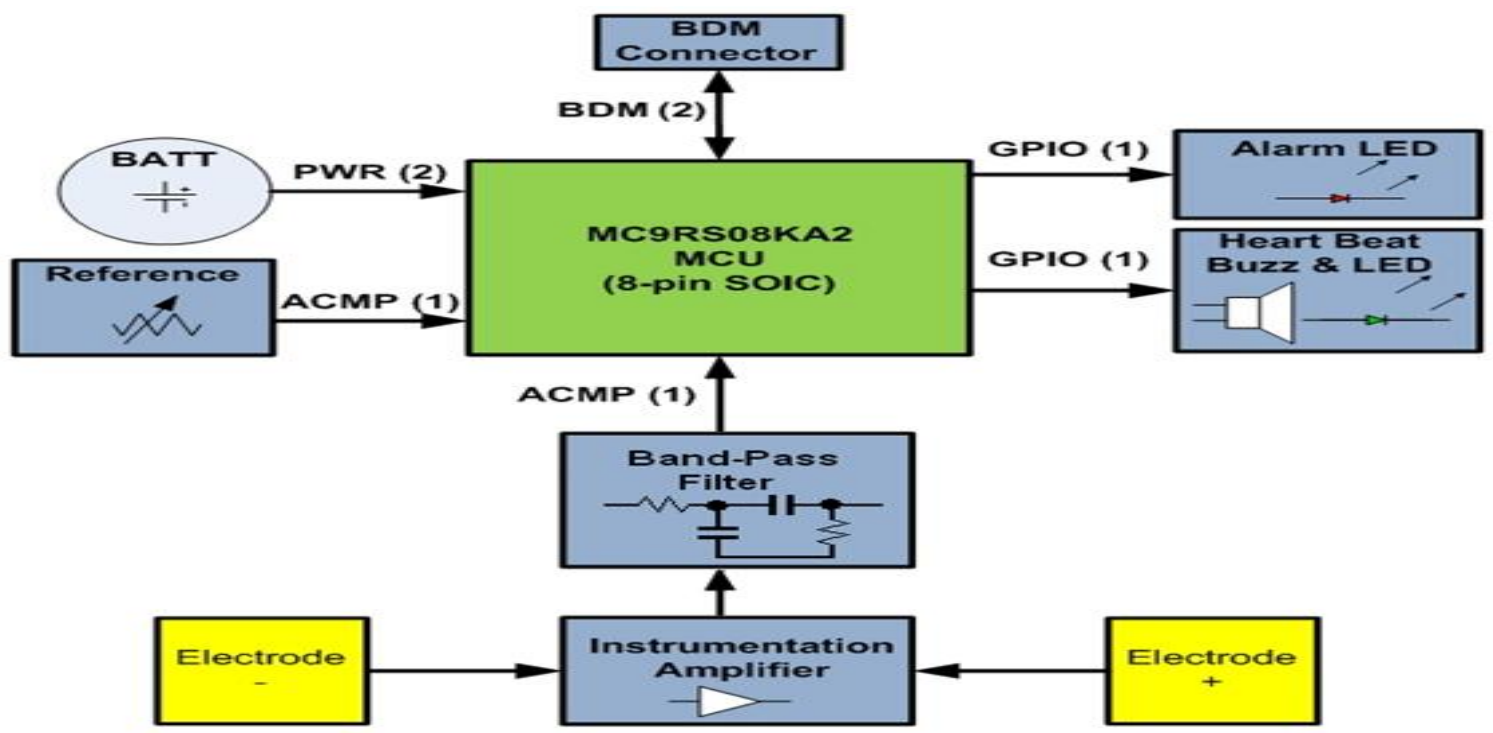

Fig.1-Heart rate monitor implementation using finger tips 


\section{II.}

THE STRUCTURE OF THE HARDWARE

The system is mainly made up of two sub- systems : patient physical states data acquisition and communication system based on wireless transceiver module and remote monitoring station ,it is shown in fig. 2 The main function of the system include : On the basis of keeping the patient movement intact, the main physical states and movement parameters of patient can be continuously monitored and recorded real - time with wireless sensors and the examiner can analyze the trend of the patient with the physical

parameters. The doctor can watch the parameter's change as graph or numeric on graphical LCD display and analyze these data to get more information about the patient, thus the examiner can know the physical states or movement parameters of patient, and then it is helpful to get the correct diagnosis result of examiner. The system can collect sensitive health information remotely and has a good applied prospect in remote healthy hare field.

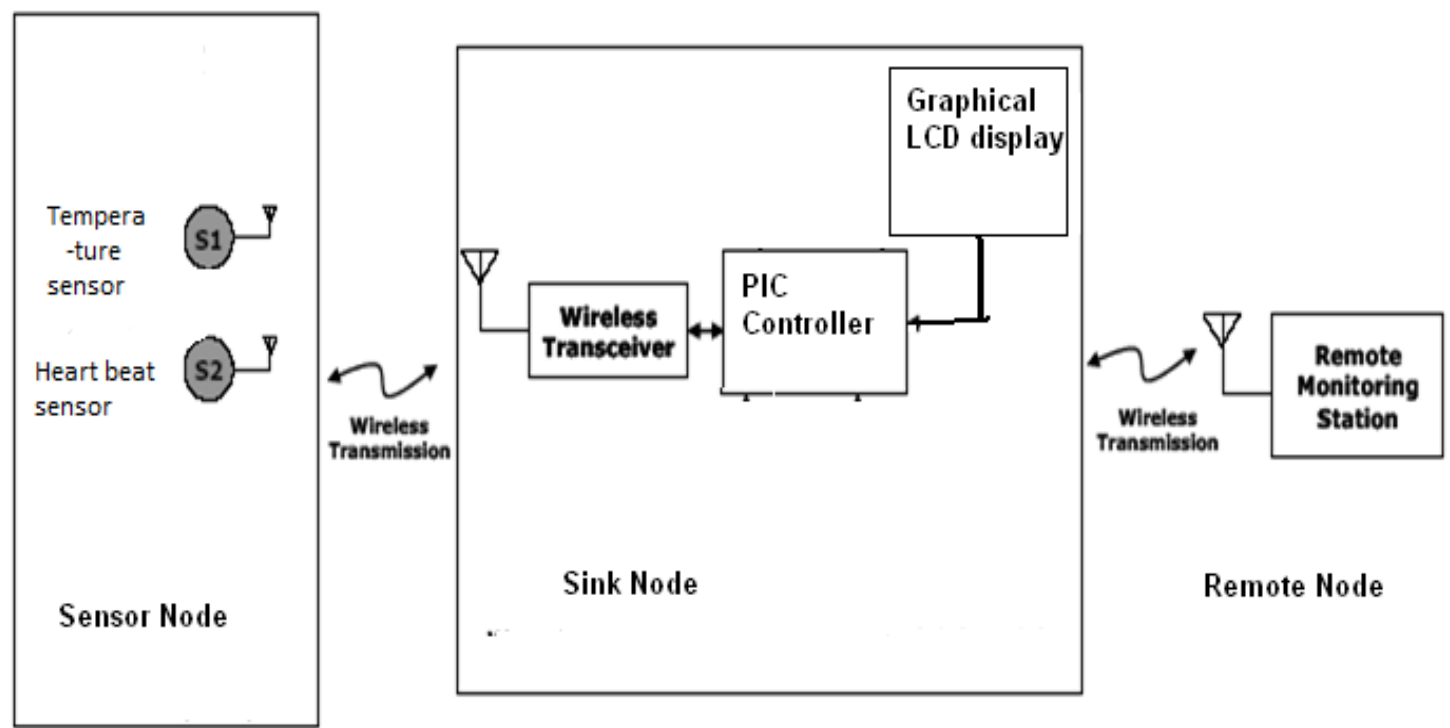

Fig. 2 overall architecture of embedded biomedical data acquisition system consisting of sensor node,sink node and remote node.

\section{PHYSICAL STATES ACQUISITION SYSTEM FOR PATIENT}

To keep the moment of the patient intact with the sensors on his body, the wireless sensors are required to be minimized and portable. Base on wireless transceiver module communication technology the patient physical states data acquisition system is made up of sensors, signal conditioning unit, A/D converter, embedded PIC controller unit and transceiver communication module. According to the state of an illness of the patient, the sensors which are fixed on patient body may be of some of these sensors ECG sensors, blood pressure sensors. Each sensor is fasted on corresponding position of the patients body[8], [9].

The hardware structure of this system is shown in figure3. The physical states and movement parameters are acquired with sensor's at first and then the signal is been transferred to signal conditioning unit to be amplified and filtered, A/D converter transforms the analogue signal to digital signal the main function of PIC controller is to integrate the data with the definition frame format, at last, the transceiver communication module sends it to remote monitoring system or display. . IEEE 802.15.4 is selected as the wireless transmission standard because of its short-range, low-cost, and low-power characteristics.

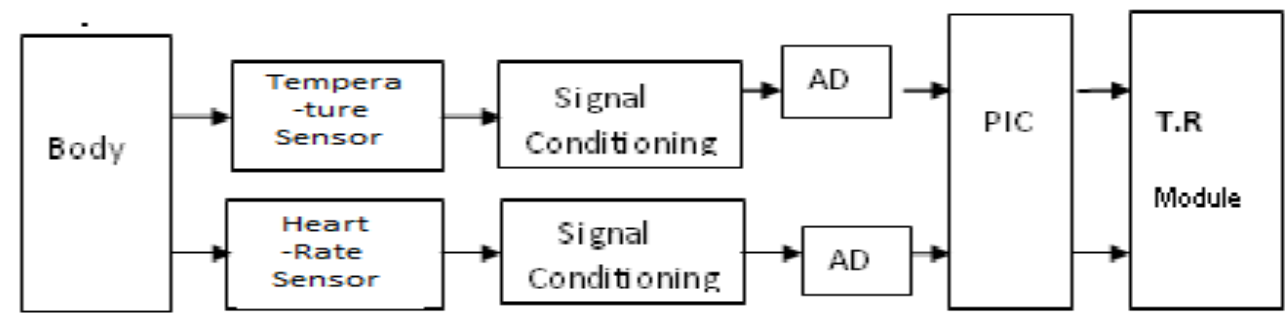

Fig . 3 The Hardware Structure of Embedded Biomedical data Acquisition System. 


\subsection{TEMPERATURE SENSOR}

Temperature is one of the fundamental physical variables in most chemical and process control applications. Accurate and reliable measurement of the temperature is important in nearly all process control applications. Temperature sensors can be analog or digital. Some of the most commonly used analog temperature sensors are: thermocouples, resistance temperature detectors (RTDs) and thermistors. Digital sensors are in the form of integrated circuits. The choice of a sensor depends on the accuracy, the temperature range, speed of response, thermal coupling, the environment (chemical, electrical, or physical) and the cost. A popular voltage output analog integrated circuit temperature sensor is the LM35DZ, manufactured by National Semiconductor. This is a 3-pin analog output sensor which provides a linear output voltage of $10 \mathrm{mV}^{0} / \mathrm{C}$. The temperature range is ${ }^{0} \mathrm{C}$ to $+100{ }^{\circ} \mathrm{C}$, with an accuracy of $\pm 1.5^{\circ} \mathrm{C}$.

\subsection{HEART BEAT SENSOR}

The Heart Beat signal is obtained by LED and LDR combination. Pulses form hands interrupts the Light reaching the LDR and this signal is read by microcontroller, The RF signal is transmitted by transmitter in a digital format. This circuit uses Manchester encoding to avoid a long trail of one or zero. The protocol is well defined for different device types ensuring compatibility with your whole entertainment system 5 bit address and 6 bit command length. Constant bit time of $1.778 \mathrm{~ms}$ bits are of equal length of $1.778 \mathrm{~ms}$ in this protocol, A logical zero is represented by a pulse in the first half of the bit time. A logical one is represented by a pulse in the second half of the bit time.

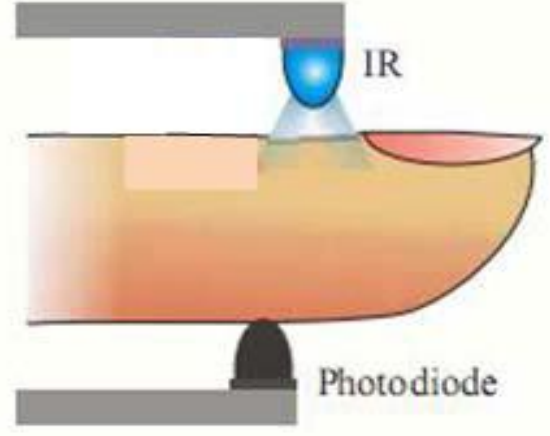

\section{Transmittance}

Fig . 4-Illustration of fingertip sensor

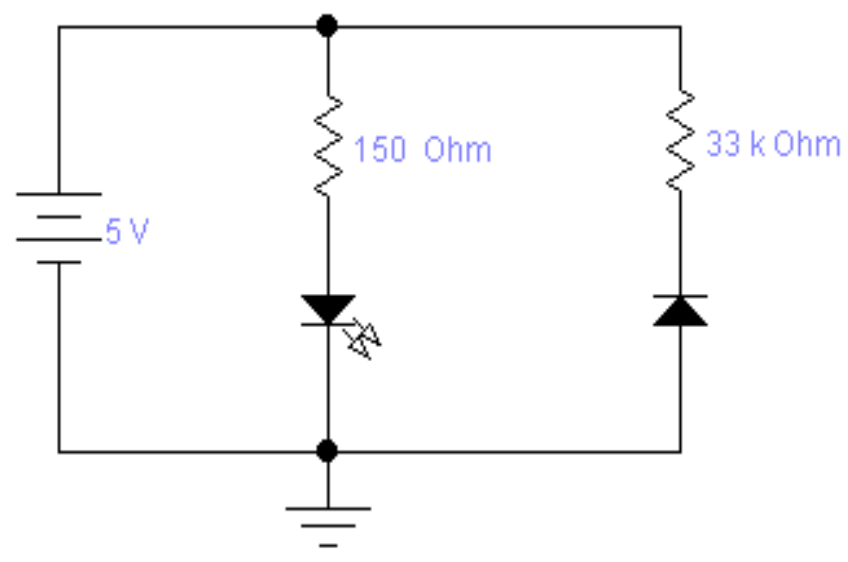

Fig . 5-The fingertip sensor circuit

\subsection{GPS RECEIVER SYSTEM}

This is a high gain GPS Receiver (5V Serial) with 4pin. Receiver is made with third generation POT (Patch Antenna on Top) GPS module. The built in 3V3 to 5V level convertor enable us to interface with normal $5 \mathrm{~V}$ Microcontrollers. The 4 Pins are 5V, TX, RX, and GND. Yes, there is no setting required, just plug in to the power (5v), your data (NMEA 0183) is ready at TX pin! This is a standalone 5V GPS Module and requires no external components .It is built with internal RTC Back up battery. It can be directly connected to Microcontroller's UART. With the use high gain GPS engine providing a solution that high position and speed accuracy performances as well as high sensitivity and tracking capabilities in urban conditions \& provides standard NMEA0183 strings in "raw" mode for any microcontroller. The module provides current time, date, latitude, longitude, speed, altitude and travel [12].

\subsection{GSM MODEM}

GSM/GPRS Modem-TTL (5V is built with Tri-band GSM/GPRS engine, works on frequencies EGSM $900 \mathrm{MHz}$, DCS $1800 \mathrm{MHz}$ and PCS $1900 \mathrm{MHz}$ It is very compact in size and easy to use as plug in module. The Modem is coming with 5V TTL interface, which allows you to connect directly to $5 \mathrm{~V}$ microcontroller. The baud rate is configurable from 9600-115200 through AT command. The GSM/GPRS TTL Modem is having internal TCP/IP stack to enable you to connect with internet via GPRS. It is suitable for SMS as well as DATA transfer application in M2M interface. You need only two wires (Tx, Rx) except Power supply to interface with microcontroller. Using this modem, you can send SMS, data and read SMS through simple AT command .Inbuilt Powerful TCP/IP protocol stack for internet data transfer over GPRS. Input Voltage: 9-12VDC [12]. 


\subsection{SIGNAL CONDITIONING}

Signal conditioner converts the output of the sensor/transducer into an electrical quantity suitable for operation of the display or recording system. Signal conditioner may vary in complexity from a simple resistance network or impedance matching device to multi-stage amplifiers and other complex electronic circuits. Signal Conditioning usually include functions such as amplification, filtering ( analog or digital ), analog to digital and digital to analog conversion or signal transmission circuitry. They help in increasing the sensitivity of instruments by amplification of the original signal or its transuded form. The buffer amplifier, which is usually an instrumentation amplifier is proposed in this paper which provides impedance buffering, signal gain and common mode rejection. It has a high input impedance, 100 Mohms or more to reduce the effects of any signal distortion. The high input impedance also minimizes errors due to the finite on resistance of the A / D convertor or channel switches. The use of programmable gain amplifiers removes the necessity to standardize on the analog input ranges.

\subsection{A/ D CONVERTOR}

The A/ D convertor carries out the process of analog to digital conversion. An A/ D convertor is a single chip integrated circuit having a single input connection for the analog signal and multiple pins for digital output. It may have $8,12,16$, or even more output pins, each representing an output bits. The higher the number of bits the higher the precision of conversion. Each step represents a change in the analog signal: 8-bits gives 256 steps, 12- bits provides 4096 steps and we get 32768 steps with 16 output bits. Speed of an A/D convertor is generally expressed as its conversion time, i. e. the time elapsed between application of a convert command and the availability of data at its output. The speed of A/ D Convertor is measured by its settling time for a full scale digital input change.

\subsection{PIC CONTROLLER}

The PIC 16F877 is an 8-bit microcontroller, which has an on-chip eight channel 10-bit Analog-toDigital Converter (ADC).First we detect fall down using accelerometer and fed to the I2C ports. The amplified and conditioned Heart Rate signal is fed to input port RB0 (INT) of the microcontroller. Also, upon command, the microcontroller reads the temperature sample stored in the RAM of the LM35 through the ADC port RA0. It is then converted and stored in the PIC16F877 memory as two 8-bit unsigned integers (0-255). After completion of signals acquisition, the microcontroller constructs the SMS messages and packs the data samples in these messages to the desired length, then communicates with the mobile phone using at-commands on its GSM modem port to send the message(s). A complete system can therefore be built using one MCU chip and a few I/O devices such as a keypad, display and other interfacing circuits. Most of the pins are for input and output, and arranged as 5 ports: PORTA (5pins), PORTB (8pins), PORTC (8pins), PORTD (8pins) and PORTE (3 pins), total of $32 \mathrm{I} / \mathrm{O}$ pins [5], [14].

\subsection{LCD DISPLAY}

The Model JHD 162A Series LCD is the typical standard HD44780 type of LCD with 16characters $\mathrm{x} 2$ row LCD module. Since this project the Heart Rate, temperature, adders and contact no to display; therefore, a LCD module is necessary.

\section{WIRELESS MONITORING OF THE SUBJECT}

Monitoring and control is the core of the real- time monitoring system for patient physical states, and it can dispose, display, save ,query and analyze the data from each patient. To know the physical states of inpatient, the physical parameters need to be monitored real -time. With the increase in the number senior citizens and chronic diseases, the number of elderly patients who need constant assistance has increased. One key point of all critical care for elderly patient is the continuous monitoring of their vital signs. The results prove that the mobility, usability and performance of our proposed system have impacts on the user's attitude, and there is a significant positive relation between the user's attitude and the intent to use our proposed system. This proposed system is expected to monitor the electrical activity of heart of the patient under critical care more conveniently and accurately for diagnosing which can be interfaced with PIC 16F877 to bring it under a network system widely for the doctor to monitor the patients condition sitting in his own office without being physically present near to the patients bed. Wireless - networked embedded device includes signal conditioning circuitry, sensors and a PIC controller with a wireless Transceiver module( CC2500). To measure or monitor human movements or activities, a graphical LCD display is selected for its low price, small size, capability of continuous measurement, and ease of integration. IEEE 802.15.4 is selected as the wireless transmission standard because of its short-range, low-cost, and low power characteristics. This system can not only realize accurate measurement of indicators, but also save the patients travel between home and the hospital. 


\section{MEASURING THE ECG SIGNAL WITH THE OSCILLOSCOPE}

The following figures show the waveforms of the obtained signal in each part of the analog front end. Figure 6 shows the waveform at output of the instrumentation amplifier. The peak-to-peak voltage of the heart signal is in the order of about $650 \mathrm{mV}$, and with noise added.

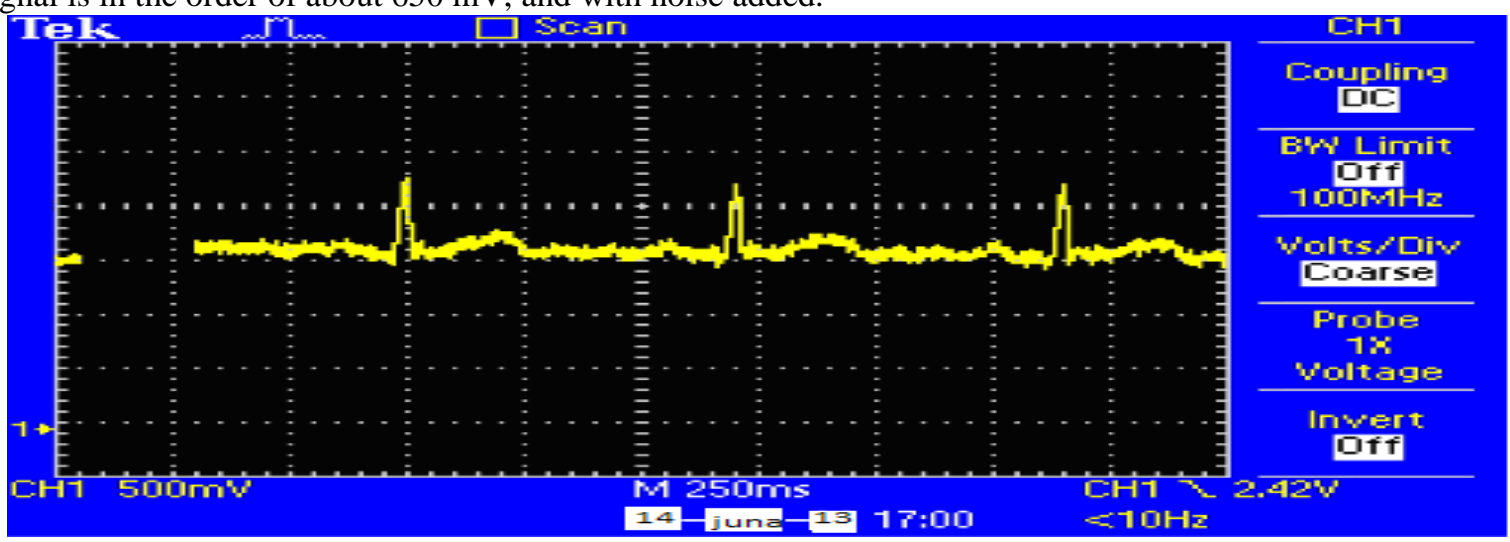

Fig . 6- Heart signal at the output of the instrumentation amplifier

Figure 7 shows the heart signal at the output of the passive band-pass filter. The noise present in the signal has been reduced; however, the peak-to-peak amplitude has been also attenuated due to filter effects. To counteract this effect and condition the signal to values of rail-to-rail voltages of the ADC, a second amplification applied using an operational amplifier.

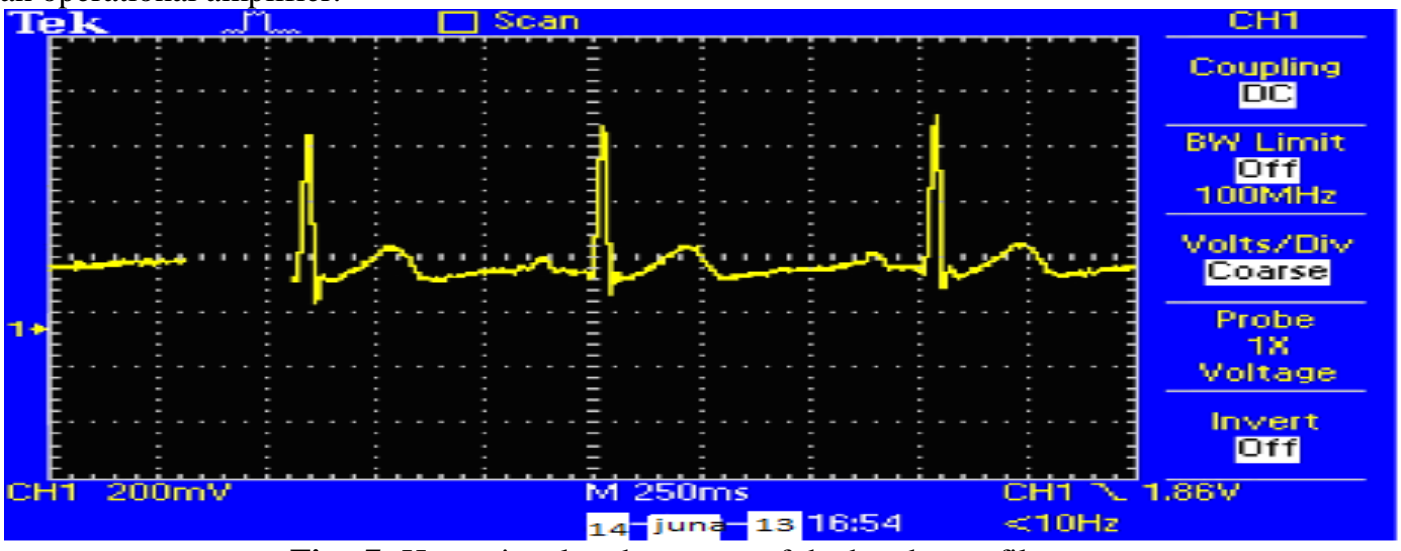

Fig . 7- Heart signal at the output of the band-pass filter

The signal at the output of the operational amplifier is shown in Figure 8 The signal has been amplified, and its peak-to-peak voltage is in the order of $3 \mathrm{~V}$. This is s optimal value to be passed to the ADC

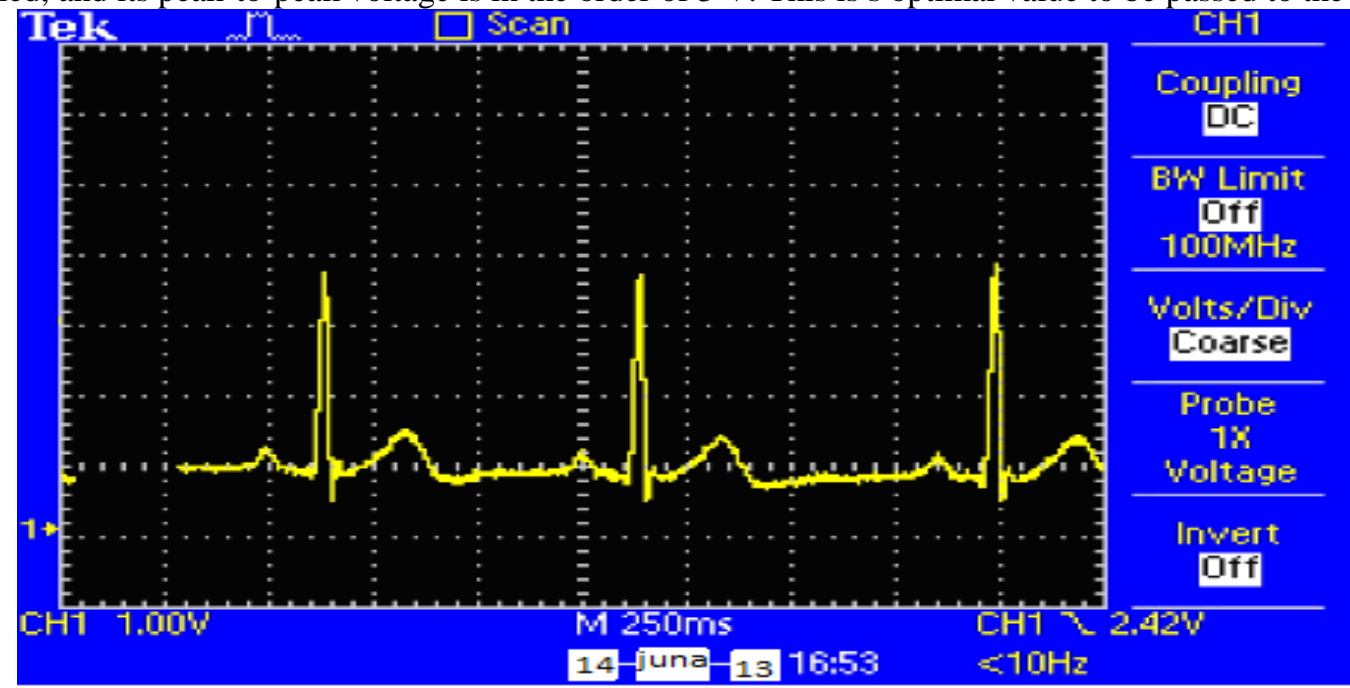

Fig . 8-Heart signal at the output of the secondary operational amplifier 


\section{CONCLUSION}

The research and design of embedded pulse monitoring instrument overcome the shortcoming of traditional pulse diagnosis system. The instrument has simple structure stable and reliable operation, high Accuracy, low power consumption, good portability full featured function, and extensive application occasion. The real time monitoring system for cardiac patient physical state is based on wireless transceiver module technology. It can be taken by patient and keep the patient moment intact because it is miniature and portable .The system can monitor and record the physical states and moment parameters real time, and the provide axuilary means for the correct diagnosis of doctor. With intelligent transreciver module, the sign of acute disease for patient can be found early, and then the patient can be helped in time, the sudden death of patient can be avoided. The wireless transreciver module technology can be suited for short distance communication, and the transmission distance is limited only about 10 meters, and then It can be suitable for in- patient monitoring. The system is important to be applied to patient care.

\section{REFERENCES}

[1]. M.M. A. Hashem, Rushdi Shams, Md. Abdul Kader, and Md. Abu Sayed, Design and Development of a Heart Rate Measuring Device using Fingertip, Department of Computer Science and Engineering, Khulna University of Engineering \& Technology (KUET), Khulna 9203, Bangladesh, 2010.

[2]. S.Allender, V.Peto, P.Scarborough, A.Boxer and M.Rayner, Coronary heart disease statistics, , British

[3]. Health Promotion Research Group, Heart Foundation Department of public health, University of Oxford,

[4]. 2007.

[5]. Mohamed Fezari, Mounir Bousbia-Salah, and Mouldi Bedda, "Microcontroller Based Heart Rate Monitor", The International Arab Journal of Information Technology, Vol. 5, No. 4, 2008.suited for short distance communication, and the transmission distance is limited only about 10 meters, and then It can be suitable for in- patient monitoring. The system is important to be applied to patient care.

[6]. U. Patel, C. Babbs, A computer based automated telephonic system to monitor patient progress in home setting, Jornal of Medical Systems, vol. 16(2), 1992, 101-112.

[7]. G. Coyle, L. Boydell, L. Brown, Home telecare for the elderly,Journal of Telemedicine and. Telecare, vol. 1, 1995, 183-185.

[8]. P. Giovas, D. Papadoyannis, D. Thomakos, et al., Transmission of electrocardiograms from a moving ambulance, Journal of Telemedicine and Telecare, vol. 4, 1998, 5-7.

[9]. Rizzi Maria,etc. " A wireless sensor network for security systems adopting the biuetooth technology", WSEAS Transactions on circuits and systems, vol. 5,no.5, 2006, p652-657

[10]. Joshua Proulx, Ryan Clifford, Sarah Sorensen, Dah-Jye Lee, and James Archibald. Development and evaluation of a Bluetooth EKG monitoring sensor. In Proceedings of the IEEE International Books: Symposium onComputer-Based Medical Systems (CBMS), 2006.

[11]. G. Bodic, "Mobile messaging technologies and services", (West Sussex, England: John Wiley\& Sons, 2005).

[12]. S. Guthery, M. Cronin, Mobile Application Development with SMS and the SIM Toolkit (New York, NY: McGraw-Hill , 2002). 